\title{
Cardio-Protective Effects of Multiport Antegrade Cold Blood Cardioplegia Versus Antegrade Cold Blood Cardioplegia in Patients With Left Ventricular Systolic Dysfunction Undergoing Conventional Coronary Artery Bypass Grafting
}

Muhammad Ali ${ }^{1}$, Muhammad Moeen ${ }^{1}$, Iftikhar Paras ${ }^{1}$, Waqas Hamid ${ }^{2}$, Saadat Khan ${ }^{3}$, Muhammad Hamid Chaudhary ${ }^{1}$

1. Cardiac Surgery, Chaudhry Pervaiz Elahi Institute of Cardiology, Multan, PAK 2. Cardiac Surgery, Sheikh Zayed Medical College/Hospital, Rahim Yar Khan, PAK 3. Echocardiography, Tabba Heart Institute, Karachi, PAK

Corresponding author: Muhammad Ali, ali.cardiacsurgeon@gmail.com

\section{Abstract \\ Introduction}

The aim of this study was to compare the in-hospital outcomes of multiport antegrade cold blood cardioplegia through vein grafts versus conventional antegrade cold blood cardioplegia in patients with left ventricle systolic dysfunction who underwent coronary artery bypass grafting (CABG).

\section{Methods}

This prospective, randomized clinical study was comprised of patients undergoing on-pump CABG at the Ch. Pervaiz Elahi Institute of Cardiology in Multan, Pakistan from November 18, 2018 to December 17, 2019. Patients with multivessel coronary artery disease and left ventricular systolic dysfunction (ejection fraction $<50 \%$ ) were included. In Group A ( $=73$ ), multiport antegrade cold blood vein graft cardioplegia was given after every distal anastomosis completed for myocardial preservation. In Group B $(\mathrm{N}=73)$, conventional antegrade cold blood cardioplegia was given for myocardial preservation.

\section{Results}

Spontaneous rhythm (without defibrillation applied) after cross-clamp removal was higher in Group A than in Group B $(93.3 \%$ vs. $85.2 \%$, p < 0.05). Duration of support, ventilation time, and hospital stay were also significantly lower in Group A than in Group B with $p=0.00001, p=0.03$, and $p=0.002$, respectively. Intraaortic balloon pump insertion ( $4.1 \%$ vs. $23.0 \%, \mathrm{p}=0.02)$ and operative mortality $(0.5 \%$ vs. $4.0 \%, \mathrm{p}=0.35)$ were also lower in Group A than in Group B. Postoperative left ventricular ejection fraction (LVEF) increased more in Group A than in Group B, and the postoperative LVEF mean value was $44.68 \%$ in Group A versus

Received 08/18/2020 Review began 08/19/2020 Review ended 08/31/2020 Published 09/08/2020

(c) Copyright 2020 Ali et al. This is an open access article distributed under the terms of the Creative Commons Attribution License CC-BY 4.0., which permits unrestricted use, distribution, and reproduction in any medium, provided the original author and source are credited. $41.26 \%$ in Group B ( $p=0.02)$.

\section{Conclusion}

Multiport vein graft blood cardioplegia provides superior myocardial protection in patients with left ventricular systolic dysfunction who underwent CABG. It is also easy to administer, so this technique can be adopted as a routine method for myocardial protection in patients with left ventricular dysfunction planned for on-pump CABG.

Categories: Cardiac/Thoracic/Vascular Surgery

Keywords: left ventricular systolic dysfunction, coronary artery bypass grafting, multiport antegrade cold blood cardioplegia

\section{Introduction}

Performing coronary artery bypass grafting (CABG) on cardiopulmonary bypass (CPB) is considered the gold standard [1]. In the United States, more than $80 \%$ of CABG procedures are performed using CPB [2]. Antegrade cardioplegia through aortic root is the most well-known method of myocardial protection. Antegrade cardioplegia provides quicker diastolic arrest and preserves myocardial function. Most cardiac centers prefer antegrade cardioplegia due to its safety, simplicity, and superiority to other methods [1]. In severely diseased coronary arteries, antegrade cardioplegia does not perfuse beyond the occluded artery, which leads to suboptimal protection to the myocardium and low ejection fraction (EF) postoperatively. Optimal myocardial protection in poor left ventricular (LV) function patients can be achieved by adequate distribution of cardioplegia to all areas of the heart and minimization of ischemia time [3,4]. Maldistribution of cardioplegia can be avoided by injection through antegrade and retrograde. Antegrade cardioplegia 
infused in the aortic root and retrograde through the coronary sinus provides marginal protection in severely occluded arteries and low LV function patients $[5,6]$. Retrograde cardioplegia has limitations that do not protect the right ventricle of the heart and introduce the risk of coronary sinus damage [6]. To address these challenges, in a severely occluded coronary artery, it is recommended to perfuse the cold blood cardioplegia in the aortic root as well as in the vein graft through multiport cardioplegia cannula, also known as the 'multiport antegrade cold blood cardioplegia' technique, which is a harmless and simple technique [7].

This study was designed to compare in-hospital outcomes of multiport antegrade cold blood cardioplegia through vein grafts versus conventional antegrade cold blood cardioplegia in patients with LV systolic dysfunction who underwent CABG.

\section{Materials And Methods}

This prospective, randomized clinical study was composed of patients undergoing CABG on CPB at the Ch. Pervaiz Elahi Institute of Cardiology in Multan, Pakistan from November 18, 2018 to December 17, 2019. Patients' informed consent signatures were collected and approved by the research evaluation unit of the College of Physicians and Surgeons of Pakistan. The study population included all patients who were admitted for CABG in the Department of Cardiac Surgery of Ch. Pervaiz Elahi Institute of Cardiology.

This study included men and women of all ages with multivessel coronary artery disease (e.g., double-vessel or triple-vessel coronary artery disease) and LV systolic dysfunction (i.e., patients with $\mathrm{EF}<50 \%$ were included). Exclusion criteria included patients with single-vessel coronary artery disease (CAD), normal biventricular systolic function (i.e., with $\mathrm{EF}>50 \%$ ), or clinically significant valvular disease (i.e., more than mild valvular disease). Patients with less than $1 \mathrm{~mm}$ target vessels or diffuse coronaries, reoperations, and a history of cerebrovascular disease were also excluded.

Randomization was applied to categorize the patients into two equal groups (Group A and Group B). Randomization was done using lottery method. We made 146 folded papers containing name of intervention (73 folded papers for each intervention) and put them in a jar. Every time before taking the patient to operating room, the staff nurse on duty was asked to pick one folded paper. The patients were randomized on the basis of folded paper chosen by the staff nurse. In Group A, multiport antegrade cold blood vein graft cardioplegia was given after every distal anastomosis was completed for myocardial preservation. In Group $\mathrm{B}$, conventional antegrade cold blood cardioplegia was given for myocardial preservation.

All CABG procedures were done by two consultant surgeons at the institute. All patients followed regular preoperative medications on the day of surgery, including bromazepam $3 \mathrm{mg}$ orally. For induction of anesthesia, morphine, midazolam, and propofol were administered, and doses were adjusted depending on patients' responses. Atracurium bromide $(1 \mathrm{mg} / \mathrm{kg})$ was injected before intubation.

In all patients, the chest was exposed through a median sternotomy. After harvesting of the left internal mammary artery and radial artery or saphenous vein conduit, $4 \mathrm{mg} / \mathrm{kg}$ of heparin was given before cannulation to maintain above 480 seconds of activated clotting time. Standard conventional CPB was established with ascending aortic cannulation, two-staged venous cannula, and regular vent-port cardioplegia cannula insertion. Hypothermia applied to $30^{\circ} \mathrm{C}$ to $32^{\circ} \mathrm{C}$. After aortic cross-clamp, $4^{\circ} \mathrm{C}$ to $8^{\circ} \mathrm{C}$ cold blood cardioplegia (blood:crystalloid cardioplegia, 4:1) was infused through the cardioplegia delivery system into the coronary arteries to arrest the heart. An initial dose of $20 \mathrm{~mL} / \mathrm{kg}$ cardioplegia was given to achieve a complete arrest.

In Group $\mathrm{A}$, after distal anastomosis of each vein graft, a free branch of a multiple perfusion set was attached to the proximal end of the vein graft for infusing the cardioplegia. A $10-\mathrm{mL} / \mathrm{kg}$ maintenance dose cardioplegia was delivered in a multiple perfusion set along with cardioplegia cannula. Until anastomosis of all distal vein grafts was completed, multiport cold blood cardioplegia was delivered simultaneously with antegrade cardioplegia. In Group B, a maintenance dose of $10 \mathrm{~mL} / \mathrm{kg}$ of cold blood cardioplegia was repeated after every graft or every 20 minutes (whichever was first or applied) through cardioplegia cannula. After cross-clamp removal, proximal anastomosis was performed after hemodynamic stability was achieved. Smooth wean off was done with or without ionotropic support. All patients were followed until one month after surgery to determine the operative outcomes.

Statistical analysis was done using IBM SPSS Statistics for Windows, Version 23.0. (Armonk, NY, IBM Corp.). Quantitative data were analyzed using means \pm standard deviation, and qualitative data were analyzed using a chi-square test. The group comparison was conducted by an independent sample t-test, and the statistical significance among the group was represented using a p value $\leqslant 0.05$.

\section{Results}

There was no significant difference among the groups regarding age, gender, diabetes, hyperlipidemia, preoperative left ventricular ejection fraction (LVEF), and preoperative creatinine kinase myocardial band (CKMB) levels $(\mathrm{p}>0.05)$. There was no difference in the number of diseased vessels, and patients in both groups had left ventricle systolic dysfunction preoperatively (Table 1). 


\section{Cureus}

\begin{tabular}{|c|c|c|c|}
\hline Characteristics & Group A Study Group ( $N=73$ ) & Group B Control Group (N = 73) & $p$ Values \\
\hline Age (years) & $53.20 \pm 8.2$ & $54.20 \pm 6.32$ & 0.41 \\
\hline Men/women & $62(84.9 \%) / 11(15.1 \%)$ & 64 (87.7\%)/9 (12.3\%) & 0.63 \\
\hline Family history (\%) & $10(13.7 \%)$ & $11(15.1 \%)$ & 0.81 \\
\hline Diabetes mellitus (\%) & $40(54.8 \%)$ & $32(43.8 \%)$ & 0.18 \\
\hline Smoking (\%) & $42(57.5 \%)$ & $50(68.5 \%)$ & 0.17 \\
\hline Hypertension & $47(64.4 \%)$ & $45(61.6 \%)$ & 0.73 \\
\hline Hyperlipidemia (\%) & $11(15.1 \%)$ & $9(12.3 \%)$ & 0.63 \\
\hline Number of diseased vessels (\%) TVD, DVD & $49(67.1 \%), 24(32.9 \%)$ & 46 (63.0\%), 27 (37.0\%) & 0.60 \\
\hline Preoperative LVEF (\%) & $38.42 \pm 6.39$ & $39.86 \pm 5.58$ & 0.14 \\
\hline Preoperative CKMB level & $20.80 \pm 10.57$ & $20.70 \pm 10.4 \varepsilon$ & 0.95 \\
\hline
\end{tabular}

\section{TABLE 1: Baseline demographic and operative characteristics}

Abbreviations: TVD, triple-vessel disease; DVD, double-vessel disease; LVEF, left ventricle ejection fraction; CKMB, creatinine kinase myocardial band.

Intra-operative data and postoperative results are shown in Table 2 . There was a highly significant difference noted between the groups regarding bypass time $(\mathrm{p}=0.001)$. In Group A (study group), the CPB time was less than that in Group B (control group).

\begin{tabular}{|c|c|c|c|}
\hline Characteristics & Group A Study Group (N = 73) & Group B Control Group (N = 73) & p Values \\
\hline Bypass time (minutes) & $118.90 \pm 29.20$ & $128 \pm 34.23$ & 0.001 (sig) \\
\hline $\mathrm{x}$-clamp time (minutes) & $62.22 \pm 14.46$ & $69 \pm 16.40$ & 0.08 \\
\hline No. of grafts (mean $\pm S D$ ) & $3.36 \pm 0.50$ & $3.31 \pm 0.55$ & 0.06 \\
\hline Postoperative CKMB level (IU) & $68.54 \pm 42.68$ & $98.62 \pm 74.63$ & 0.002 (sig) \\
\hline Duration of support (hours) & $6.11 \pm 9.17$ & $10.10 \pm 15.62$ & 0.0001 (sig) \\
\hline Ventilation time (hours) & $5.60 \pm 7.04$ & $09 \pm 25.57$ & 0.03 (sig) \\
\hline Hospital stay time (days) & $6.69 \pm 1.22$ & $6.62 \pm 2.57$ & 0.002 (sig) \\
\hline IABP (\%) & $3(4.1)$ & $13(23.0)$ & 0.02 (sig) \\
\hline Spontaneous rhythm (\%) & 93.3 & 85.2 & 0.004 (sig) \\
\hline Operative mortality (\%) & 0.5 & 4.0 & 0.35 \\
\hline Postoperative LVEF (\%) & 44.68 & 41.26 & 0.02 (sig) \\
\hline
\end{tabular}

\section{TABLE 2: Comparison of intra-operative and postoperative outcomes}

Abbreviations: SD, standard deviation; LVEF, left ventricle ejection fraction; CKMB, creatinine kinase myocardial band; IABP, intra-aortic balloon pump; IU, international unit; sig, significant difference.

Spontaneous rhythm (without defibrillation applied) after cross-clamp removal was higher in Group A than in Group B ( $93.3 \%$ vs. $85.2 \%$, p < 0.05). Duration of support, ventilation time, and hospital stay were also significantly lower in Group A than in Group B with $p=0.00001, p=0.03$, and $p=0.002$, respectively. Operative mortality $(0.5 \%$ vs. $4.0 \%, \mathrm{p}=0.35)$ and intra-aortic balloon pump (IABP) insertion $(4.1 \%$ vs. $23.0 \%$, $\mathrm{p}=0.02$ ) were also lower in Group A than in Group B. Postoperative LVEF increased more in Group A than in Group B, with a postoperative LVEF mean value of $44.68 \%$ in Group A versus $41.26 \%$ in Group B ( $p=0.02)$. Postoperative peak CKMB level was statistically considerably higher $(p=0.002)$ in Group B compared to that 


\section{Discussion}

With the progression of medical technology, percutaneous intervention methodology for the treatment for $\mathrm{CAD}$ is considered conventional therapy; however, in extreme multivessel coronary disease, patients were referred for $C A B G$. The primary principle of $C A B G$ is to provide adequate blood supply to the ischemic areas through revascularization. Hence, several improvements in cardioplegia delivery techniques have been made to improve myocardial preservation $[8,9]$. As there is a high probability of postoperative mortality in CABG due to myocardial dysfunction [10,11], myocardial protection during CPB can be achieved by providing adequate distribution of a cardioplegia solution throughout the myocardium [12]. Distribution of cardioplegia beyond the occluded coronary vessels effectively protects the myocardium [13].

To date, the combination of antegrade and retrograde cardioplegia infusion is considered the gold standard in myocardial protection [14]. Conventional cold blood antegrade root cardioplegia is considered an effective method due to its safety and simplicity.

This study method was nearly identical to the method suggested by Goldman et al., which stated that careful graft perfusion provides appropriate cooling of the most ischemic zones to protect myocardium by reperfusion of cold blood cardioplegia. The researchers concluded that this method is suitable for patients who present with acute ischemia, failed angioplasty, left main occlusion, and LV systolic dysfunction [15].

Until now, not many studies have been done on cardioplegia through vein grafts to protect the myocardium. There are conflicting results found in different studies about the present method. Onem et al. showed that there was no advantage of perfusing the antegrade cold blood cardioplegia through vein grafts in low-risk patients [13]. Other published studies demonstrated that there was no benefit to myocardium preservation when using simultaneous antegrade root and vein graft cardioplegia together. Both studies had a small sample size and excluded poor LVEF patients [16,17].

Our study results were almost the same as an earlier randomized controlled study conducted by Sher-IMurtaza et al., who reported lower cardiac troponin I levels when patients were given passive multiport cold blood cardioplegia, which indicates it is superior to conventional cardioplegia [7]. The lower bypass time, lower incidence of arrhythmia after x-clamp removal, lower IABP insertion count, lower postoperative CKMB*isoenzyme level (IU) release, and the increases in postoperative LVEF were all good signs of myocardial protection in the study group. Our study found that antegrade cold blood graft cardioplegia provided superior myocardial preservation in LV systolic dysfunction due to proper cardioplegia distribution in even proximal occluded artery.

\section{Conclusions}

Multiport vein graft infusion of blood cardioplegia provides superior myocardial protection in CABG patients having preoperative LV systolic dysfunction. Due to its ease of administration, this technique can be adopted as a routine method for myocardial protection in patients with $\mathrm{LV}$ dysfunction planned for on-pump CABG.

\section{Additional Information}

\section{Disclosures}

Human subjects: Consent was obtained by all participants in this study. Department of Academic Affairs, CPEIC Multan issued approval CPEIC-031-2019. Animal subjects: All authors have confirmed that this study did not involve animal subjects or tissue. Conflicts of interest: In compliance with the ICMJE uniform disclosure form, all authors declare the following: Payment/services info: All authors have declared that no financial support was received from any organization for the submitted work. Financial relationships: All authors have declared that they have no financial relationships at present or within the previous three years with any organizations that might have an interest in the submitted work. Other relationships: All authors have declared that there are no other relationships or activities that could appear to have influenced the submitted work.

\section{References}

1. Alvarez JM, Cooke JC, Shardey GC, Goldstein J, Harper RW: Orthodox coronary artery bypass surgery: the gold standard in surgical coronary artery disease intervention. Asia Pac Heart J. 1999, 8:148-153. 10.1016/S1328-0163(99)90051-9

2. Hussain G, Azam H, Baig MAR, Ahmad N: Early outcomes of on-pump versus off-pump coronary artery bypass grafting. Pak J Med Sci. 2016, 32:917-921. 10.12669/pjms.324.9680

3. Onorati F, Renzulli A, De Feo M, et al.: Does antegrade blood cardioplegia alone provide adequate myocardial protection in patients with left main stem disease?. J Thorac Cardiovasc Surg. 2003, 126:13451351. 10.1016/s0022-5223(03)00736-0

4. Soltesz EG, Laurence RG, De Grand AM, Cohn LH, Mihaljevic T, Frangioni JV: Image-guided quantification of cardioplegia delivery during cardiac surgery. Heart Surg Forum. 2007, 10:381-386. 
5. Sanjay OP, Srikrishna SV, Prashanth P, Kajrekar P, Vincent V: Antegrade versus antegrade with retrograde delivery of cardioplegic solution in myocardial revascularisation. A clinical study in patients with triple vessel coronary artery disease. Ann Card Anaesth. 2003, 6:143-148.

6. Borger MA, Wei KS, Weisel RD, et al.: Myocardial perfusion during warm antegrade and retrograde cardioplegia: a contrast echo study. Ann Thorac Surg. 1999, 68:955-961. 10.1016/s0003-4975(99)00797-3

7. Sher-I-Murtaza M, Rizvi HMFA, Baig MAR, Hamid W, Zaman H: Myocardial protection with multiport antegrade cold blood cardioplegia and continuous controlled warm shot through vein grafts during proximal ends anastomosis in conventional coronary artery bypass. J Pak Med Assoc. 2016, 66:53-58.

8. Shaefi S, Mittel A, Loberman D, Ramakrishna H: Off-pump versus on-pump coronary artery bypass graftinga systematic review and analysis of clinical outcomes. J Cardiothorac Vasc Anesth. 2019, 33:232-244. 10.1053/j.jvca.2018.04.012

9. Chu D, Bakaeen FG, Dao TK, LeMaire SA, Coselli JS, Huh J: On-pump versus off-pump coronary artery bypass grafting in a cohort of 63,000 patients. Ann Thorac Surg. 2009, 87:1820-1827. 10.1016/j.athoracsur.2009.03.052

10. Onorati F, De Feo M, Mastroroberto P, et al.: Determinants and prognosis of myocardial damage after coronary artery bypass grafting. Ann Thorac Surg. 2005, 79:837-845. 10.1016/j.athoracsur.2004.07.060

11. Allen BS, Buckberg GD: Myocardial management in arterial revascularization. Arterial Grafting for Coronary Artery Bypass Surgery. He GW (ed): Springer, Berlin, Heidelberg; 2006. 51-62. 10.1007/3-540-30084-8_6

12. Nissinen J, Biancari F, Wistbacka JO, et al.: Safe time limits of aortic cross-clamping and cardiopulmonary bypass in adult cardiac surgery. Perfusion. 2009, 24:297-305. 10.1177/0267659109354656

13. Onem G, Sacar M, Baltalarli A, Ozcan AV, Gurses E, Sungurtekin H: Comparison of simultaneous antegrade/vein graft cardioplegia with antegrade cardioplegia for myocardial protection. Adv Ther. 2006, 23:869-877. 10.1007/BF02850208

14. Radmehr H, Soleimani A, Tatari H, Salehi M: Does combined antegrade-retrograde cardioplegia have any superiority over antegrade cardioplegia?. Heart Lung Circ. 2008, 17:475-477. 10.1016/j.hlc.2008.04.009

15. Goldman BS, Ovil Y, Mycyk T: A technique for selective graft perfusion during aortocoronary bypass . J Card Surg. 1987, 2:495-498. 10.1111/j.1540-8191.1987.tb00206.x

16. Gürsoy M, Bakuy V, Hatemi AC: Delivering cardioplegia beyond totally occluded native coronary arteries through the saphenous bypass vein graft: is it really a protective technique?. Kosuyolu Kalp Derg. 2012, 15:100-104. 10.5578/kkd.4032

17. Hatemi AC, Ulusoy RE, Gürsoy M, et al.: Myocardial protection with simultaneous antegrade/vein graft cardioplegia compared to antegrade cardioplegia alone in elective coronary artery bypass grafting patients. Balkan Med J. 2011, 28:75-79. 10.5174/tutfd.2009.03129.1 